\title{
Dissipative-Radiative Micropolar Fluid Transport in a Non- Darcy Porous Medium with Cross-Diffusion Effects
}

\author{
Mohammad Ferdows ${ }^{1}$, MD. Shamshuddin ${ }^{2}$, Khairy Zaimi ${ }^{3, *}$ \\ Research Group of Fluid Flow Modeling and Simulation, Department of Applied Mathematics, University of Dhaka, Dhaka-1000, Bangladesh \\ Department of Mathematics, Vaagdevi College of Engineering (Autonomous), Warangal, Telangana, India \\ Institute of Engineering Mathematics, Universiti Malaysia Perlis, Pauh Putra Campus, 02600 Arau, Perlis, Malaysia
}

\section{ARTICLE INFO}

\section{Article history:}

Received 21 May 2020

Received in revised form 20 July 2020

Accepted 25 July 2020

Available online 31 July 2020

\section{Keywords:}

Porous media; micropolar fluid; viscous dissipation; diffusion effect; thermal radiation; stretching sheet

\section{ABSTRACT}

In this work, the micropolar fluid flow and heat and mass transfer past a horizontal stretching sheet through a porous medium are studied including the Soret-Dufour effect in the presence of viscous dissipation. A uniform magnetic field is applied transversely to the direction of the flow. The governing differential equations of the problem are transformed into a system of non-dimensional differential equations which are solved numerically by Nachtsheim-Swigert iteration technique along with the sixth order Runge-Kutta integration scheme. The velocity, microrotation, temperature and concentration profiles are presented for different parameters and interpreted at length. Results show that with an increase in vortex viscosity ratio parameter, suction parameter and radiation parameter, velocity is decreased whereas it increases with the increase of magnetic parameter, Darcy number and Eckert number. Angular velocity significantly elevated by increasing the suction parameter, surface nonlinearity parameter and magnetic parameter. Temperature gradient escalate with the increase of magnetic parameter and Dufour number, while a reverse trend is observed in case of increase of Darcy number, Eckert number and Soret number. Concentration gradient putrefies with Schmidt number and Dufour number. However, concentration grows with Soret number. The present problem finds significant applications in hydromagnetic control of conducting polymeric sheets and magnetic materials processing.

\section{Introduction}

Micropolar fluids, distinctly non-Newtonian in nature, are referred to those that contain microconstituents belonging to a class of complex fluids with non-symmetrical stress tensor [1]. These fluids respond to micro-rotational motions and spin inertia, and therefore can support couple stress and distributed body torque which are not achievable with the classical Navier-Stokes equations or the viscoelastic flow models [2]. The Micropolar fluid models designed by Eringen [3] are developed

\footnotetext{
* Corresponding author.

E-mail address: khairy@unimap.edu.my (Khairy Zaimi)
} 
to make an analysis of the flow characteristics of physiological fluids (blood containing corpuscles), colloidal suspensions, paints, liquid crystal suspensions, oils containing fine suspensions, industrial contaminants containing toxic chemicals, lubricants, organic/inorganic hybrid nanocomposites and clay which are fabricated by melt intercalation [4].

External convective boundary layer flows from horizontal and vertical flat stretchable sheets have been investigated quite extensively. Tough early studies explained few important flows in viscous fluid but non-Newtonian fluid like micropolar fluid has received less attention towards boundary layer flows adjacent to sheets (or indeed other geometries) as it has several applications in technological processes. Crane [5] noted that usually the stretching sheet is assumed to be inextensible, but situations may arise in the polymer industry in which it is necessary to deal with a stretching plastic sheet. For examples, materials manufactured by aerodynamic extrusion processes and heat-treated materials traveling between a feed roll and a wind-up roll or on a conveyor belt possess the characteristics of a moving continuous stretching surface. Moreover, numerous metallurgical processes occupy the system of cooling of continuous strips or filaments by drawing them through a quiescent fluid and that in the process of drawing, these strips are sometimes stretched. An important matter is that the final product depends to a great extent on the rate of cooling. By drawing such strips in an electrically conducting fluid subjected to a magnetic field, the rate of cooling can be controlled and a final product of desired characteristics can be achieved [6]. The study of heat and mass transfer is necessary for determining the quality of the final product. The boundary layer models for steady or unsteady micropolar fluids in various geometries (stationary or moving surface, linear or nonlinear stretching surface etc) with/or without heat transfer considering various flow conditions (no-slip or slip, suction/injection at the surface) and thermal boundary conditions (constant/variable surface temperature or heat flux) have extensively been studied by numerous researchers [7-21].

Ishak [22] initiated the numerical study of thermal boundary layer flow over a stretching sheet in a micropolar fluid. Later, Ahmed et al., [23] provided the numerical solution for the micropolar fluid over a non-linearly stretching sheet. Prasad et al., [24] conducted a speculative exploration to study the influence of convective heat transfer during the flow of micropolar fluid past a stretching surface. Rawat et al., [25] scrutinized the influence of multi-physical components flow in a non-Darcy porous medium through the nonlinear stretching surface. These studies have all demonstrated the significant influence of boundary surface of the stretching sheet on thermofluid dynamic characteristics in micropolar fluids. Consequently, assorted hypothetical and practical attributes of the flow and heat transfer with regards to micropolar fluids recently have been explored $[26,27]$. Also, a stable structure given by nanofluid has attracted many investigators are Eid et al., [28] did recent investigation about Carreau nanofluid flow numerically through nonlinear stretching sheet. Again Eid et al., [29] extended the Carreau nanofluid flow with chemically reactive species. AlHossainy et al., [30] described discussion about MHD nanofluid flow for external yield stress effect. Eid [31] explored the facts about non-Newtonian bio-nanofluid flow in suction/blowing process with effects of NP shapes. Lahmar et al., [32] did work about squeezing unsteady nanofluid flow under inclined magnetic field and variable thermal conductivity effects.

Moreover, the thermal-diffusion (Soret) effect, has been utilized for isotope separation, and in mixtures between gases with very light molecular weight $(\mathrm{Hz}, \mathrm{He})$ and of medium molecular weight $(\mathrm{Nz}$, air). The diffusion-thermo (Dufour) effect was found to be of a considerable magnitude such that it cannot be ignored, described by Eckert and Drake [33] in their book. Recently plenty of investigators used Soret and Dufour effects are Hayat et al., [34] (for axi-symmetrical viscous flow), Makinde and Olanrewaju [35] and Shateyi et al., [36] (for mixed convection viscous flow), Srinivasacharya and Kaladhar [37] (for couple stress fluid), Shamshuddin and Siva Reddy [38] (for free 
convection rotating micropolar fluid flow) and Shamshuddin et al., [39] (for mixed convection rotating micropolar fluid flow).

Modeling of viscous dissipation effects on a flow of a fluid-saturated porous medium was considered by several authors and they have modelled this effect in different ways. Darcy's law was agreed by most of the authors. But till now various authors [40-42] are taking only one term with velocity derivative for viscous dissipation effect in Darcy medium which is wrong. Recently, AlHadhrami [43] proposed a new model for viscous dissipation in a porous medium which is probably adequate for most practical purposes, which motivated us to undertake and extend the studies of Rehman et al., [20] and Bhargava et al., [44]. Motivated by the above-referenced work and the numerous possible industrial applications of the problem, it is of paramount interest in this study in order to clarify the parametric behavior of the flow of free convection of a micropolar fluid over a horizontal stretching sheet in the presence of dynamic effects of viscous dissipation, thermaldiffusion, and diffusion-thermo.

\section{Problem Formulation}

We consider the isothermal, steady, laminar, hydromagnetic free convection flow of an incompressible micropolar fluid flowing past a stretching sheet coinciding with the plane $y=0$, the flow being confined in the region $y>0$. The flow configurations and the coordinate system are shown in Figure 1. The flow under consideration is also subjected to a strong transverse magnetic field $B_{0}$ with a constant intensity along the $y$ - axis. Two equal and opposite forces are introduced along the $x$-axis so that the surface is stretched keeping the origin fixed.

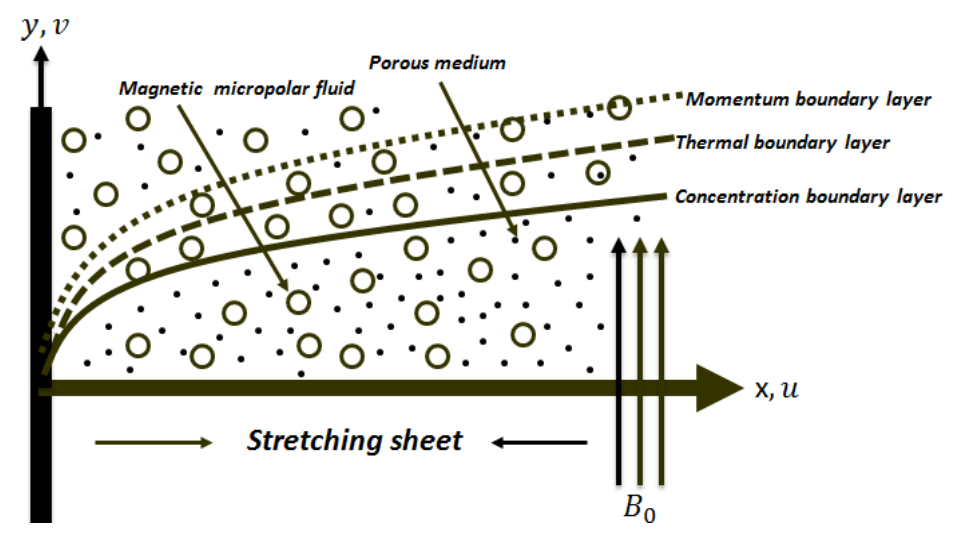

Fig. 1. Schematic diagram of flow configuration

It is assumed that the velocity of a point on a sheet is proportional to its distance from the slit. All the fluid properties are assumed to be isotropic and constant. Under the usual boundary layer and Boussinesq approximations, the governing equations for the problem under consideration can be written as (Rehman et al., [20], Bhargava et al., [44])

$$
\frac{\partial u}{\partial x}+\frac{\partial v}{\partial y}=0
$$

$$
u \frac{\partial u}{\partial x}+v \frac{\partial u}{\partial y}=\left(v+\frac{S}{\rho}\right) \frac{\partial^{2} u}{\partial y^{2}}+g \beta\left(T-T_{\infty}\right)-\frac{\sigma B_{0}^{2}}{\rho} u+\frac{S}{\rho} \frac{\partial N}{\partial y}-\left(v+\frac{S}{\rho}\right) \frac{u}{k_{p}}
$$




$$
\begin{aligned}
& u \frac{\partial N}{\partial x}+v \frac{\partial N}{\partial y}=\frac{v_{S}}{\rho j} \frac{\partial^{2} N}{\partial y^{2}}-\frac{S}{\rho j}\left(2 N+\frac{\partial u}{\partial y}\right) \\
& u \frac{\partial T}{\partial x}+v \frac{\partial T}{\partial y}=\frac{\kappa}{\rho c_{p}} \frac{\partial^{2} T}{\partial y^{2}}-\frac{\partial q_{r}}{\partial y}+\frac{D_{m} K_{T}}{c_{s} c_{p}} \frac{\partial^{2} C}{\partial C^{2}}+\frac{1}{c_{p}}\left(v+\frac{S}{\rho}\right)\left[\frac{u^{2}}{k}+\left(\frac{\partial u}{\partial y}\right)^{2}\right] \\
& u \frac{\partial C}{\partial x}+v \frac{\partial C}{\partial y}=D_{m} \frac{\partial^{2} C}{\partial y^{2}}+\frac{D_{m} K_{T}}{T_{m}} \frac{\partial^{2} T}{\partial y^{2}}
\end{aligned}
$$

In Eq. (2) the Darcian porous drag force term is defined by the term, $-\left(v+\frac{S}{\rho}\right) \frac{u}{k_{p}}$, which is linear in terms of the translational velocity, $u$. With $S=0$, the micropolar effects disappear and this term reduces to the conventional Newtonian Darcy drag force i.e. $-v \frac{u}{k_{p}}$. The micro-rotation component, $N$, is coupled to the linear momentum Eq. (2) via the angular velocity gradient term, $\frac{S}{\rho} \frac{\partial N}{\partial y}$. A coupling exists between the translational velocity components, $u$ and $v$, in Eq. (3) via the convective acceleration terms, $u \frac{\partial N}{\partial x}$ and $v \frac{\partial N}{\partial y}$. Furthermore, there is a second coupling term linking the angular velocity with the $x$-direction velocity gradient in Eq. (3), $-\frac{S}{\rho j}\left(2 N+\frac{\partial u}{\partial y}\right)$. The microrotation viscosity (or spin-gradient viscosity) $v_{S}$ is defined by $v_{S}=\left(\mu+\frac{S}{2}\right) j[26]$. We note that in the viscous shear diffusion term, $\left(v+\frac{S}{\rho}\right) \frac{\partial^{2} u}{\partial y^{2}}$, the Newtonian kinematic viscosity is now supplemented by the Eringen micropolar vortex viscosity, $S$. In the present work, it is assumed that the micro-inertia per unit mass $j$ is constant. Also, positive or negative $n$ indicates the acceleration and deceleration of the sheet from the extruded slit respectively. The last term on the right-hand side of the energy equations Eq. (4) signifies the viscous dissipation effect in Darcy media through porous media, which is modelled according to the theory proposed by Al-Hadhrami et. al., [43]. Thermal radiation is simulated using the Rosseland diffusion approximation Sparrow [45] and in accordance with this the radiative heat flux $q_{r}$ is given by

$q_{r}=-\frac{4 \sigma_{1}}{3 \kappa_{1}} \frac{\partial T^{4}}{\partial y}$

where $\sigma_{1}$ is the Stefan-Boltzmann constant and $\kappa_{1}$ is the mean absorption coefficient. Unexpressed in the Rosseland diffusion model is a unidirectional radiative flux and the condition is $\frac{\partial q_{r}}{\partial y}>>\frac{\partial q_{r}}{\partial x}$. This model is valid for optically thick media in which thermal radiation propagates only a limited distance prior to experiencing scattering or absorption [46]. The local thermal radiation intensity is due to radiation emanating from proximate locations in the vicinity of which emission and scattering are comparable to the location of interest. For zones where conditions are appreciably different, 
thermal radiation has been shown to be greatly attenuated before arriving at the location under consideration. The energy transfer depends on conditions only in the area adjacent to the plate regime i.e. the boundary layer regime. It is assumed that the temperature differences within the flow are sufficiently small such that $T^{4}$ may be expressed as a linear function of temperature. This is accomplished by expanding $T^{4}$ in a Taylor series about $T_{\infty}$ and neglecting higher-order terms, thus

$T^{4} \cong 4 T_{\infty}^{3} T-3 T_{\infty}^{4}$

The appropriate boundary conditions suggested by the physical conditions are

(i) On the plate surface

$u=U_{w}=B x^{n}, v=v_{0}(x), N=-S \frac{\partial u}{\partial y}, T=T_{w}\left(=T_{\infty}+A x^{\gamma}\right), C=C_{w}\left(=C_{\infty}+D x^{\gamma}\right)$ at $y=0$,

(ii) matching with the quiescent free stream

$u \rightarrow U_{\infty}, N \rightarrow 0, T \rightarrow T_{\infty}, C \rightarrow C_{\infty}$ as $y \rightarrow \infty$,

where the subscripts $w$ and $\infty$ refer to the wall and boundary layer edge, respectively. The relationship between the microrotation function $N$ and the surface shear $\partial u / \partial y$ is chosen for investigating the effect of different surface conditions for the microrotation of the micropolar fluid elements. The conditions are generally of importance in micropolar boundary layer analysis. When microrotation parameter $S=0$, we obtain $N=0$ which represents not able to rotate (Rehman et al., [20]). Finally, $A, D$ and $\gamma$ are the constants.no-spin condition i.e. the microelements in a concentrated particle flow-close to the wall are

\section{Similarity Solutions}

The partial differential equations Eq. (1)-(5) are transformed into non-dimensional form by mean of following dimensionless variables (see Anwar Beg et al., [47])

$$
\left.\begin{array}{l}
\eta=y\left[\frac{B(n+1)}{2 v}\right]^{\frac{1}{2}} x^{\frac{n-1}{2}}, u=B x^{n} f^{\prime}(\eta), \quad v=-\left[B v\left(\frac{n+1}{2}\right)\right]^{\frac{1}{2}} x^{\frac{n-1}{2}}\left[f+\frac{n-1}{n+1} \eta f^{\prime}\right] \\
N=B\left[\frac{B(n+1)}{2 v}\right]^{\frac{1}{2}} x^{\frac{3 n-1}{2}} h(\eta), \quad \theta(\eta)=\frac{T-T_{\infty}}{T_{w}-T_{\infty}}, \quad \phi(\eta)=\frac{C-C_{\infty}}{C_{w}-C_{\infty}}
\end{array}\right\}
$$

Substituting Eqs. (6), (7) and (10), into Eqs. (1)-(5), produces the following ordinary differential equations

$$
(1+\Delta) f^{\prime \prime \prime}+f f^{\prime \prime}-\frac{2}{(n+1)}\left(M+\frac{(1+\Delta)}{D a}\right) f^{\prime}-\frac{2}{(n+1)} n f^{\prime 2}+\Delta h^{\prime}+\frac{2}{(n+1)} G r \theta=0
$$




$$
\begin{aligned}
& \left(1+\frac{\Delta}{2}\right) h^{\prime \prime}-\frac{2}{(n+1)} \Delta \frac{1}{\xi}\left(2 h+f^{\prime \prime}\right)-\frac{3 n-1}{n+1} h f^{\prime}+h^{\prime} f=0, \\
& \frac{1}{P n} \theta^{\prime \prime}+\theta^{\prime} f-\frac{2}{n+1} \gamma \theta f^{\prime}+(1+\Delta) E c\left[f^{\prime \prime 2}+\frac{2}{(n+1)} \frac{1}{D a} f^{\prime 2}\right]+D u \phi^{\prime \prime}=0, \\
& \phi^{\prime \prime}+\operatorname{Sc} \phi^{\prime} f-\frac{2}{(n+1)} S c \gamma f^{\prime} \phi+S c \operatorname{Sr} \theta^{\prime \prime}=0,
\end{aligned}
$$

with the corresponding boundary conditions

$$
\left.\begin{array}{l}
f=f_{w}, \quad f^{\prime}=1, \quad h=-S f^{\prime \prime}, \quad \theta=1, \quad \phi=1 \text { at } \eta=0 \\
f^{\prime} \rightarrow 0, \quad h \rightarrow 0, \quad \theta \rightarrow 0, \quad \phi \rightarrow 0 \text { as } \eta \rightarrow \infty
\end{array}\right\} .
$$

Here, the primes denote differentiation with respect to $\eta$ (non-dimensional $y$-coordinate) and $\Delta=\frac{S}{\mu}$ is the vortex viscosity parameter, $G r=\frac{g \beta\left(T_{w}-T_{\infty}\right) x}{U_{w}^{2}}$ is the local Grashof number, $M=\frac{\sigma B_{0}^{2} x}{\rho U_{w}}$ is the local magnetic parameter and $B_{0}=\frac{B}{\sqrt{x}}$ is the magnetic field. Darcy number is defined as $D a=\frac{k_{p}}{x^{2}}, \xi=\frac{j U_{w}}{v x}$ is the micro-inertia density parameter and Prandtl number is written as $\operatorname{Pr}=\frac{\rho v c_{p}}{\kappa} . \quad R=\frac{\kappa \kappa_{1}}{4 \sigma_{1} T_{\infty}^{3}}$ is the radiation parameter, which is defined in $P n=\frac{3 R \operatorname{Pr}}{3 R+4}$, $E c=\frac{U_{w}^{2}}{c_{p}\left(T_{w}-T_{\infty}\right)}$ is the Eckert number, $D u=\frac{D_{m} K_{T}\left(C_{w}-C_{\infty}\right)}{v c_{s} c_{p}\left(T_{\infty}-T_{\infty}\right)}$ is the Dufour number, $S c=\frac{v}{D_{m}}$ is the Schmidt number, $\operatorname{Sr}=\frac{D_{m} K_{T}\left(T_{w}-T_{\infty}\right)}{T_{m}\left(C_{w}-C_{\infty}\right)}$ is the Soret number and $f_{w}=-v_{0}(x)\left[B v\left(\frac{n+1}{2}\right)\right]^{-\frac{1}{2}} x^{-\frac{n-1}{2}}$ is the suction parameter are local similarity parameters which are required in the nondimensionlization of the model. This is a perfectly valid approach which has been used by many scientists including heat transfer and magnetofluid dynamics. It retains the requisite validity of the physical and solutions presented in our paper are locally independent of information from other streamwise locations as noted by Sparrow and Yu [48] and Pantokratoras [49].

\subsection{Skin Friction, Rate of Heat and Mass Transfer}

The parameters of engineering interest for the present problem are the skin friction coefficient $\left(C_{f}\right)$, plate couple stress $\left(M_{w}\right)$, local Nusselt number $(N u)$ and Sherwood number $(S h)$, which indicate physically the wall shear stress, couple stress, the rate of heat transfer and the local surface mass flux, respectively. The dimensionless skin-friction coefficient, couple stress, Nusselt number, and Sherwood number for impulsively started plate are given by 


$$
\begin{aligned}
& C_{f}=\frac{\tau_{w}}{\frac{1}{2} \rho U^{2}}=2 \sqrt{\frac{(n+1)}{2 \operatorname{Re}}} f^{\prime \prime}(0), \\
& M_{w}=\frac{j\left(\mu+\frac{S}{2}\right)}{\frac{1}{2} \rho v U_{w}}\left(\frac{\partial N}{\partial y}\right)_{y=0}=\left(1+\frac{\Delta}{2}\right) \xi(n+1) h^{\prime}(0), \\
& N u=-\left.\frac{x}{\left(T_{w}-T_{\infty}\right)} \frac{\partial T}{\partial y}\right|_{y=0}=-\sqrt{\frac{\operatorname{Re}(n+1)}{2}} \theta^{\prime}(0), \\
& S h=\left.\frac{x}{\left(C_{w}-C_{\infty}\right)} \frac{\partial C}{\partial y}\right|_{y=0}=-\sqrt{\frac{\operatorname{Re}(n+1)}{2}} \phi^{\prime}(0),
\end{aligned}
$$

where Re is the Reynolds number, and hence the values proportional to the skin-friction coefficient, couple stress, Nusselt number and Sherwood number are $f^{\prime \prime}(0), h^{\prime}(0),-\theta^{\prime}(0)$ and $-\phi^{\prime}(0)$, respectively.

\section{Numerical Computation}

The numerical solutions of the non-linear differential equations Eq. (11) to (14) under the boundary conditions (15) are performed by applying a shooting method namely Nachtsheim and Swigert [50] iteration technique (guessing the missing values) along with sixth-order Runge-Kutta iteration scheme. A step size of $\Delta \eta=0.01$ is chosen to satisfy the convergence criterion of $10^{-6}$ in all cases. The value of $\eta_{\infty}$ is found in each iteration loop by $\eta_{\infty}=\eta_{\infty}+\Delta \eta$. The maximum value of $\eta_{\infty}$ to each group of parameters $\Delta, n, M, G r, D a, f_{w}, \xi, \operatorname{Pr}, R, E c, S, S c, \gamma, D u$ and $S r$ is determined, when the values of the unknown boundary conditions at $\eta=0$ do not change to successful loop with error less than $10^{-6}$. In order to verify the effects of the step size $\Delta \eta$, the computations were performed for the model with three different step sizes as $\Delta \eta=0.01$, $\Delta \eta=0.005$ and $\Delta \eta=0.001$. As shown in Figures 2-5 the differences between various cases are minimal.

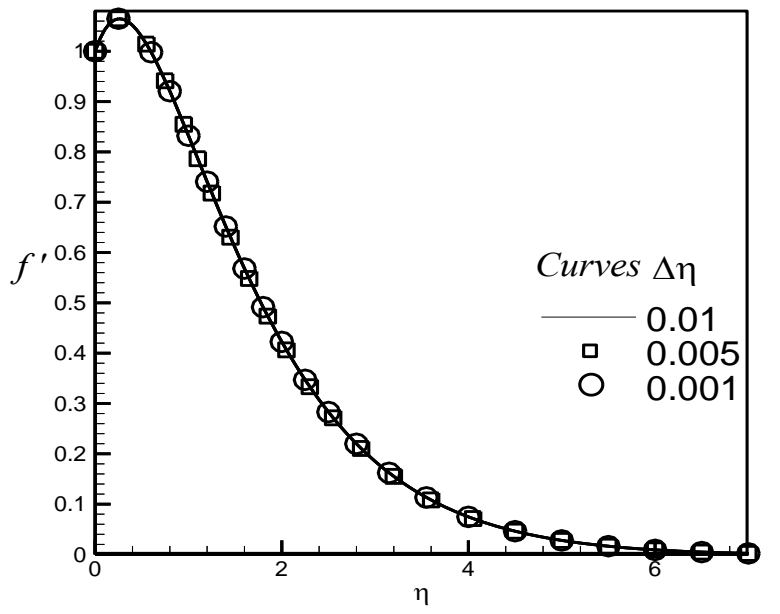

Fig. 2. Distribution of velocity for different values of $\Delta \eta$

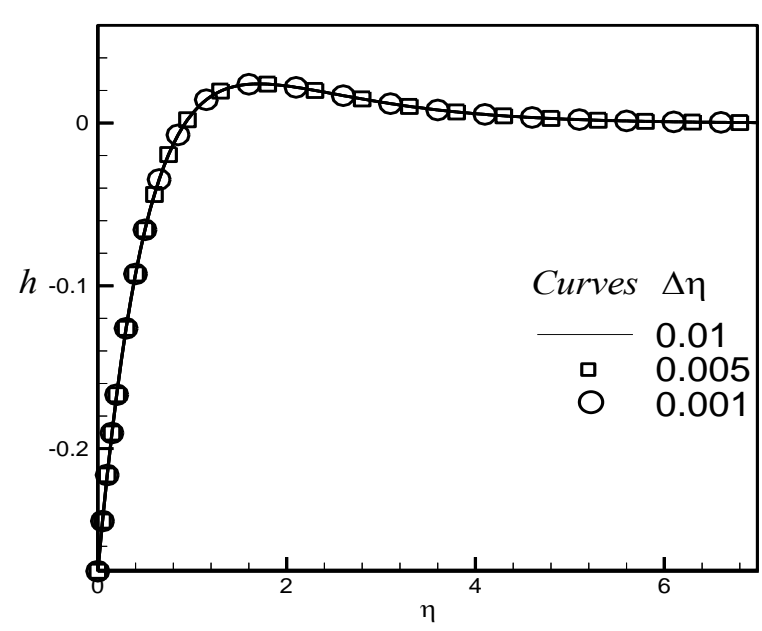

Fig. 3. Distribution of microrotation for different values of $\Delta \eta$ 


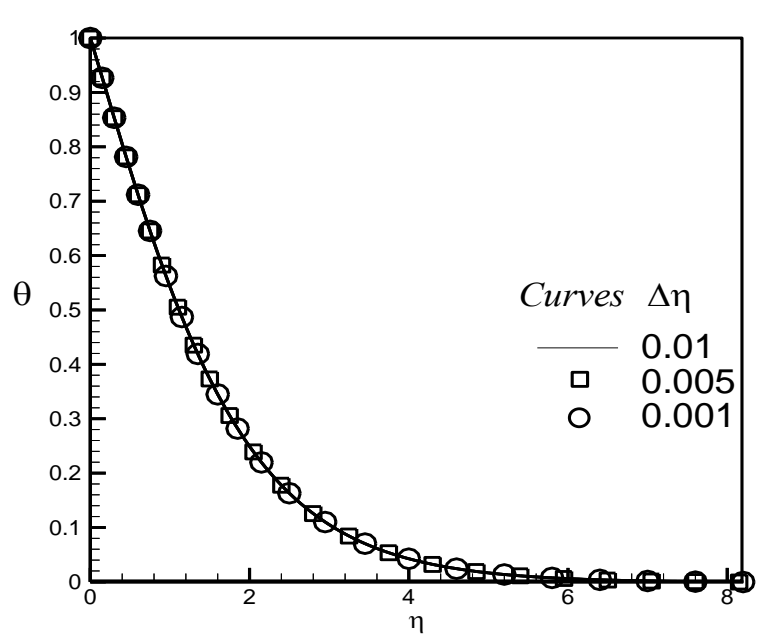

Fig. 4. Distribution of temperature for different values of $\Delta \eta$

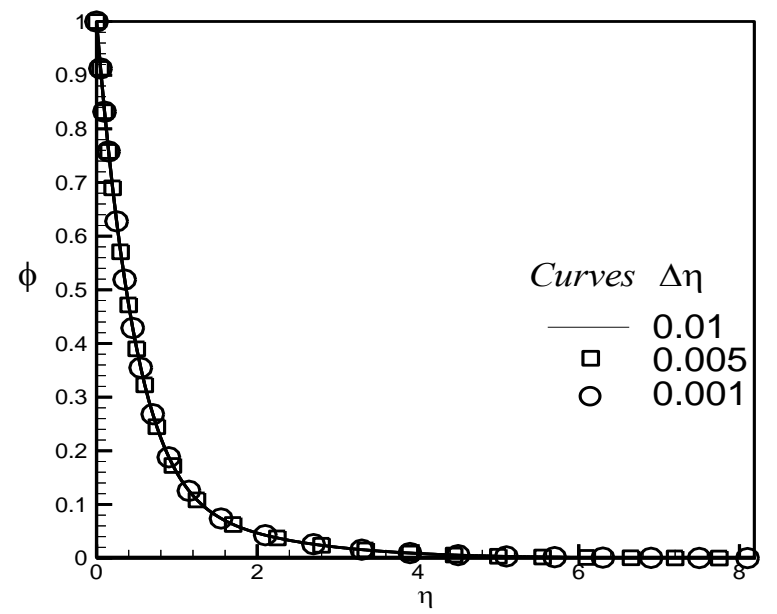

Fig. 5. Distribution of concentration for different values of $\Delta \eta$

\section{Results and Discussion}

For the purpose of discussing the results of the flow field represented in Figure 1, the numerical computations are presented in the form of non-dimensional velocity, angular velocity, temperature and concentration profiles in Figures 6-37. We set the $n=1.0, D a=0.003, G r=5.0, \Delta=0.2, M=3.0$, $R=3.0, \gamma=0.02, E c=0.3, D u=0.5, S c=0.5, S r=0.5, f w=0.5 \quad$ values of parameter unless otherwise mentioned in specific figures/tables. Figures 6-8 demonstrate the impact of micropolar material parameter or the vortex viscosity ratio parameter on translational velocity, microrotation and temperature contours across the flow characteristics. The micropolar parameter, $\Delta=S / \mu$ symbolize the ratio of Eringen vortex viscosity to Newtonian kinematic viscosity. When $\Delta=0$ vortex viscosity vanishes $(\mu \rightarrow 0)$ and the micropolar gas reduces to a Newtonian gas. For $\Delta>0$ with progressively larger values of $\Delta$, the concentration of micro-elements is exalted significantly, and spinning motions will be inhibited. It is seen that the translational velocity distribution (see Figure 6) is greater for a feeble micropolar fluid $(\Delta=0.2)$ as compared with a study micropolar fluid $(\Delta=1.0)$ . Micro-polarity therefore perpetually induces acceleration near the sheet and afterward, it shows escalation nature far away from the sheet. Figure 7 shows that the magnitude of microrotation velocity at the wall is also strongly intensified as $\Delta$ increases. The microrotation profiles grow consistently from the wall to the free stream, i.e. the peak magnitude in microrotation (angular velocity) is always attained at the wall irrespective of the value of $\Delta$. This is due to the presence of an increasing concentration of microelements which enhances vortex viscosity. A similar trend is also observed in the case of temperature profiles (Figure 8). It is observed that slowly and gradually temperature rises as $\Delta$ increases.

Figures 9-12 show the effect of suction on the distributions of linear momentum, microrotation, temperature, and concentration, respectively. It can be inspected from Figure 9 that the velocity diminishes with an increasing value of $f_{w}$ and hence slow down the boundary layer flow. Physically, the increase of $f_{w}$ indicates that $v_{0}<0$ which gives the mass transfer at the sheet due to suction. Also, the velocity of the fluid is more at $\eta=0$ for larger values of $f_{w}$. As $\eta \rightarrow \infty$ the velocity of the fluid converges to the boundary conditions at $\eta=8$. Here the case of $f_{w}>0$ is considered throughout the study. The presence of suction causes adherence of the boundary layer to sheet surface whereas blowing results in the opposite effect. In the case of suction, a markedly lower linear 
velocity is computed (Figure 9). The removal of micropolar fluid via the sheet pores destroys momentum in the boundary layer. Figure 10 indicates that the response in microrotation to $\eta-$ coordinate. Here suction is found to accelerate the microrotation. In general, injection or blowing induces weak acceleration in the spin of microelements. However, behaves reversely further from the wall where injection decelerates the microrotation.

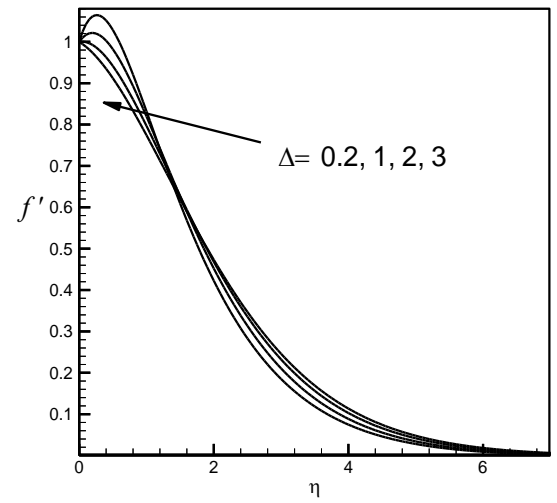

Fig. 6. Distribution of velocity for different values of $\Delta$

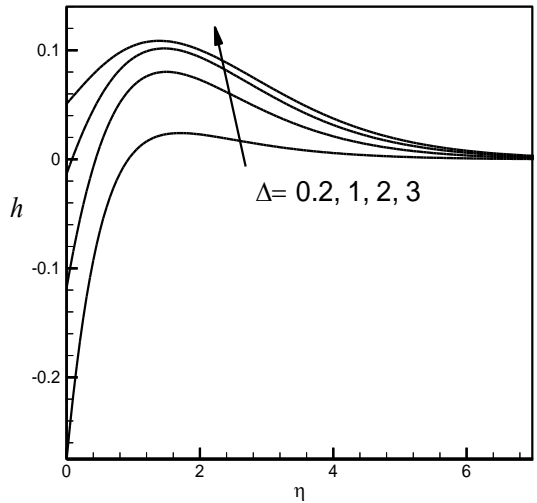

Fig. 7. Distribution of microrotation for different values of $\Delta$

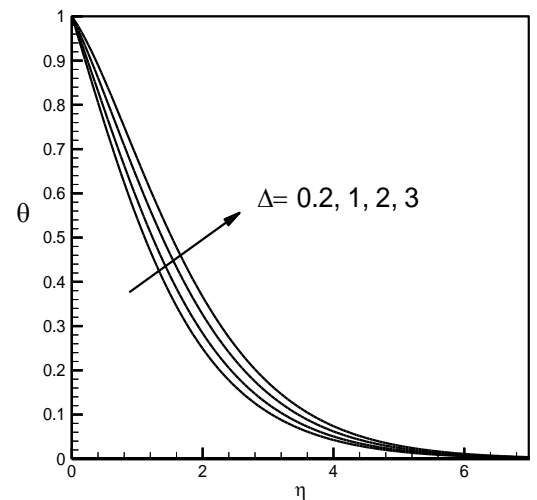

Fig. 8. Distribution of temperature for different values of $\Delta$

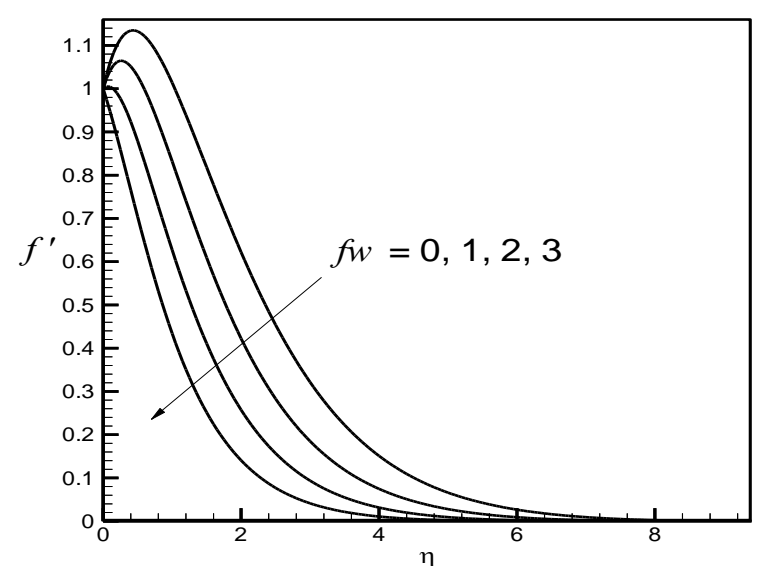

Fig. 9. Distribution of velocity for different values of $f_{w}$

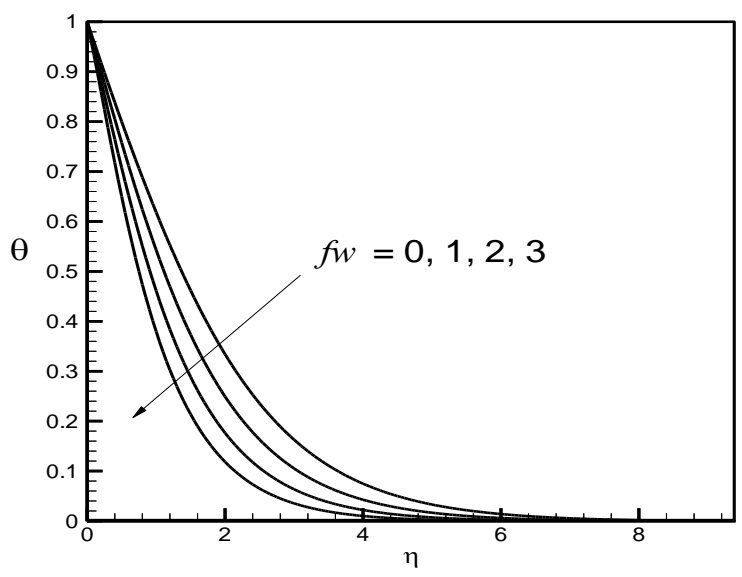

Fig. 11. Distribution of temperature for different values of $f_{w}$

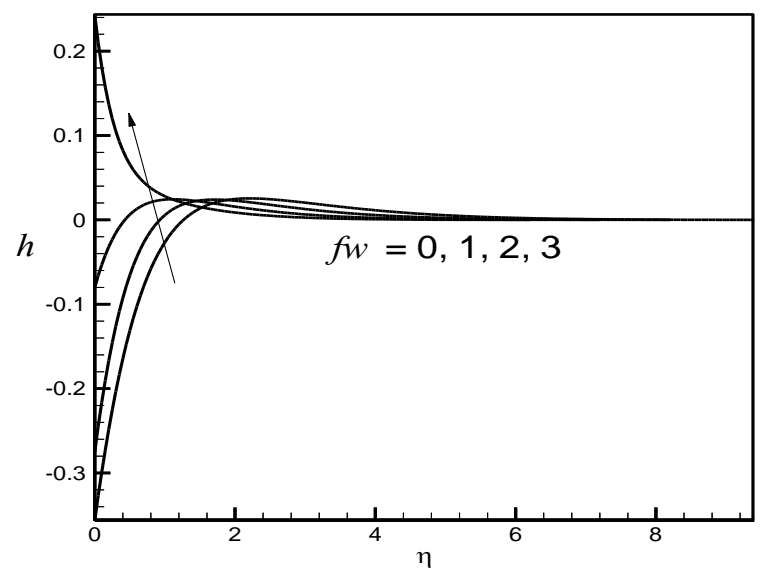

Fig. 10. Distribution of microrotation for different values of $f_{w}$

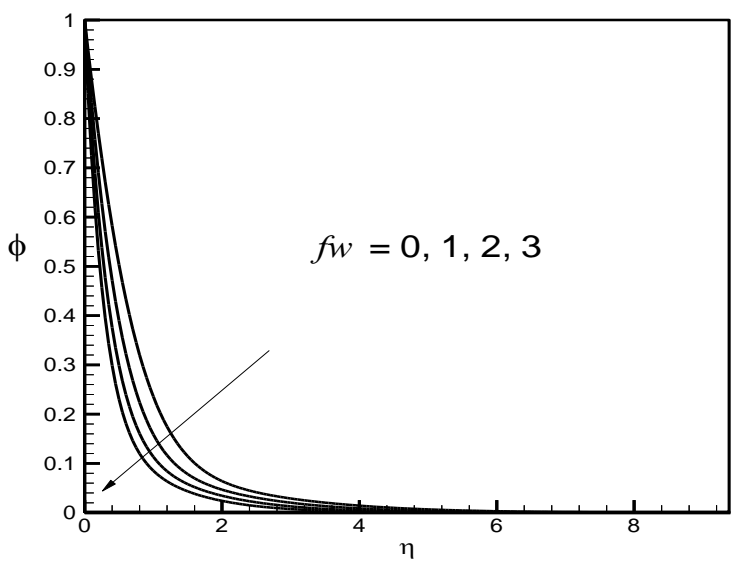

Fig. 12. Distribution of concentration for different values of $f_{w}$ 
From this, it is clearly revealed that microelements are space-dependent and depending on the location of the mass flux wall condition exerts a different influence. This is the reason for angular velocity accelerates in case of suction. Figures 11-12 exhibits that both temperature and concentration of the fluid is suppressed with wall suction. The associated thermal and solutal boundary layer thickness are therefore both decreased with grater suction effect.

Figures 13-16 display the micropolar gas flow characteristics with various nonlinear stretching parameter $(n)$. This parameter arises throughout all the boundary layer equations Eq. (11)-(14). Generally, with greater positive $n$ values, corresponding to deceleration of the stretching wall. Hence velocity distribution is decreased (Figure 13). Acceleration in microrotational profiles is marked with an increasing $n$ value (Figure 14). It is found from Figure 15 that temperature contours increase for the case of fluid suction. It is also noticeable that for fluid suction temperature profiles do not crossover away from the stretching sheet and increases with an increase of $n$. Uniformly moving surface represents $n=0$. Figure 16 portrays the effect of nonlinear stretching parameter on the concentration. The same trend occurred in temperature is seen in concentration as the sheet is being stretched $n>0$ i.e. concentration gradient increases with an increase in $n$. All the flow characteristics peak near the sheet. However, after some distance from the sheet the influence of $n$ values are reversed. Once again asymptotically smooth decays to the free stream are achieved for all distributions.

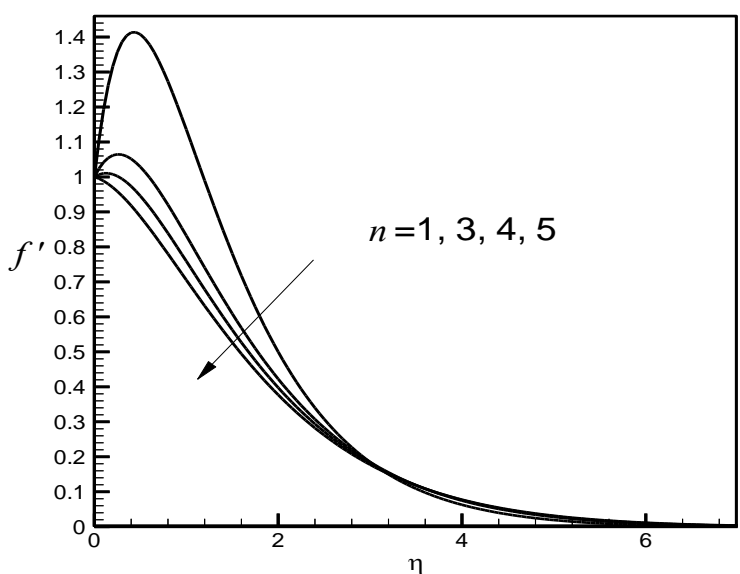

Fig. 13. Distribution of velocity for different values of $n$

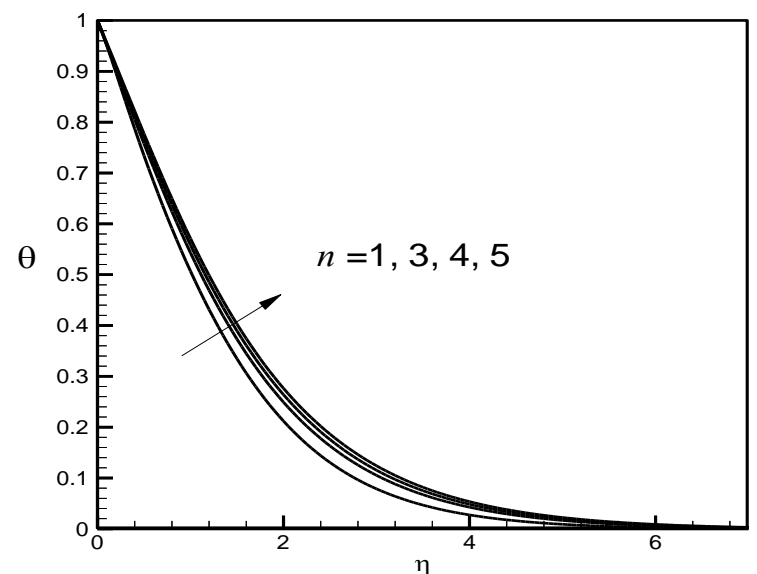

Fig. 15. Distribution of temperature for different values of $n$

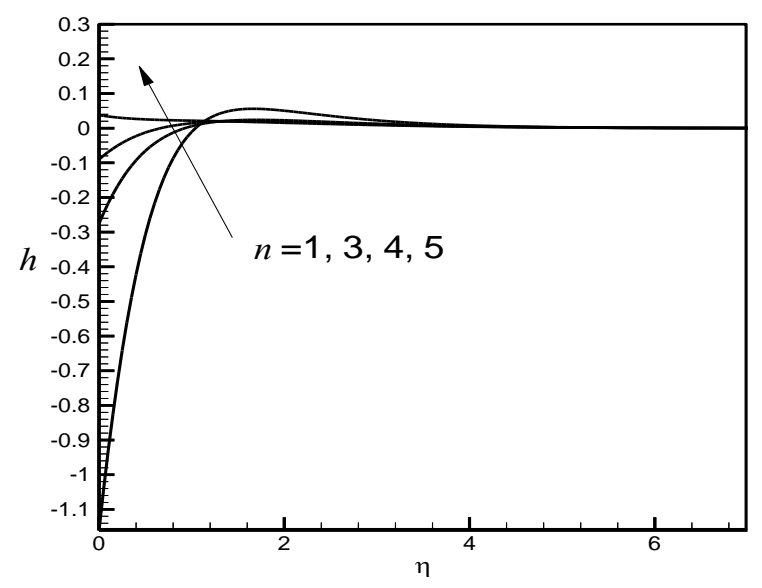

Fig. 14. Distribution of microrotation for different values of $n$

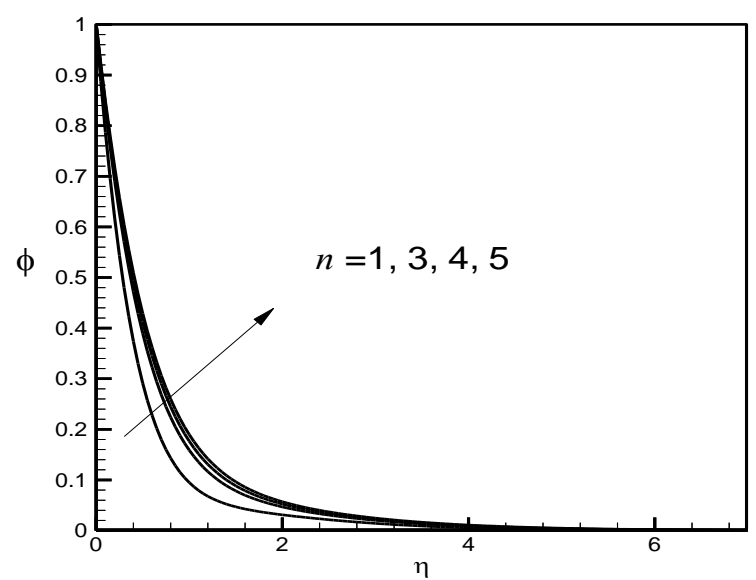

Fig. 16. Distribution of concentration for different values of $n$ 
Figures 17-19 represent the effects of magnetic field parameter $(M)$ on velocity, angular velocity and temperature. This parameter has evidently a suppressive effect on the velocity and angular velocity (microrotation) profiles as shown in Figures 17 and 18. Micro-rotation and velocity are strongly damped with greater positive magnetic field parameter $(M)$ owing to the Lorentzian drag force components, which causes a reduction in the fluid velocity. Lesser regulation (i.e. acceleration) of the velocity flow and spin of micro-elements is therefore attained with a stronger magnetic field. It is noted that despite the inhibiting effect of magnetic field on the velocity field, flow reversal is never induced (i.e. the velocity remains positive even with the very strong magnetic field). The classical velocity shoot near the generator wall is also captured in the computations as is seen in Figure 17. Overall the implications for magnetohydrodynamic generator performance is that subtle adjustments can be achieved in performance via the modification in the transverse magnetic field since $M$ is linearly directly proportional to $B_{0}^{2}$. Asymptotically smooth solutions are obtained for all $M$ values as shown by the gradual modest of all profiles with large $\eta$ values. This confirms the prescription of an adequately large infinity boundary condition. The enhancement in temperatures (Figure 19) with positive $M$ is attributable to the dissipation in the kinetic energy expended in dragging the micropolar gas against the action of the transverse magnetic field (for $M>0$ the field is inhibiting, for $M<0$ it is assistive). Additional force manifests in the addition of the thermal energy to the gas, which causes a rise in temperature.

Figures 20-22 depict the variations of velocity, angular velocity component and temperature with buoyancy parameter $(G r) . G r$ is taken to be positive to represent cooling of the sheet by free convection currents. It arises in the buoyancy term, $G r \theta$, in the momentum equation. It has a profound influence on the thermo-fluid dynamics processes due to linear in nature. Figure 20 shows that velocity is amplified substantially near the boundary. At a critical distance from the wall, however, the influence of increasing $G r$ becomes inhibitive. It decelerates the velocity and this is maintained into the free stream. The influence of free convection currents decays further from the wall and this leads to retardation in the flow. Grembodied the relative influence of the thermal buoyancy force to the viscous hydrodynamic force. For $G r>1$ the thermal buoyancy effect dominates viscous effects. Figure 21 reveals that although initially there is weak retardation in the angular velocity component, $h$, a sudden rise in profiles is noticed with higher values of $G r$. Since Grashof number becomes assistive to the spin of microelements-h values are markedly elevated throughout the majority of the boundary layer regime and attain asymptotically a non-zero value in the free stream. Peak microrotation however consistently arises quite soon in the boundary layer. The temperature function $(\theta)$ is reduced near the wall with greater $G r$ values. Thermal boundary layer thickness is therefore decreased.

Figures 23, 24 present the evolution of micropolar fluid flow characteristics with Darcy number $(D a)$. Velocity profiles reveal that initially although there is weak acceleration. Later velocity attains increasing nature and becomes assistive to the higher values of $D a$ and pointedly exalted throughout the majority of the boundary layer regime. This is due to diminishing in Darcian drag force via procuring values of permeability of flow regime. Hence, the exceptional permeability of porous media builds the fluid flow while deceleration in micropolar fluid flow is attained subject to very lesser values of permeability. Temperature (Figure 24) is strongly inheritance with grater $D a$ throughout the flow regime. The flow control is attained via a porous medium because of the large values of $D a$ yields an exceptional reduction in temperature contours. The thermal energy inhibits from the surface of the sheet towards the flow field. This will reduce the temperature of the flow field and hence the thickness of the thermal boundary layer decreases. 


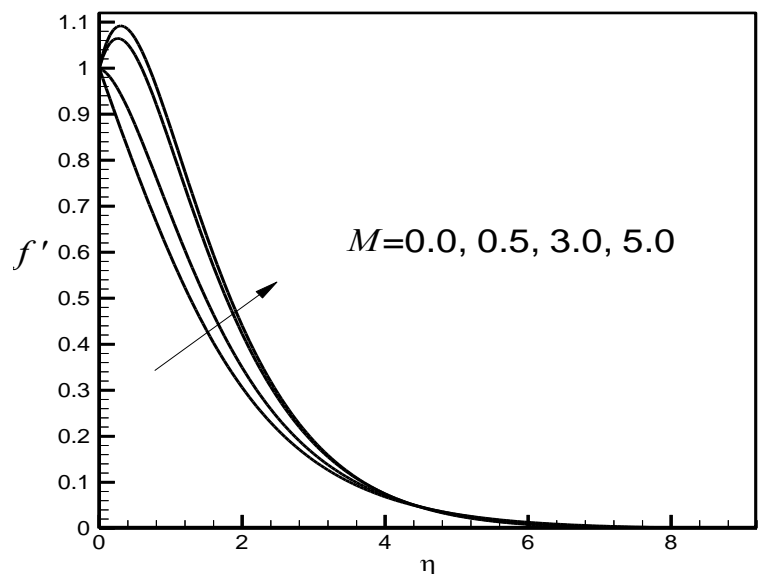

Fig. 17. Distribution of velocity for different values of $M$

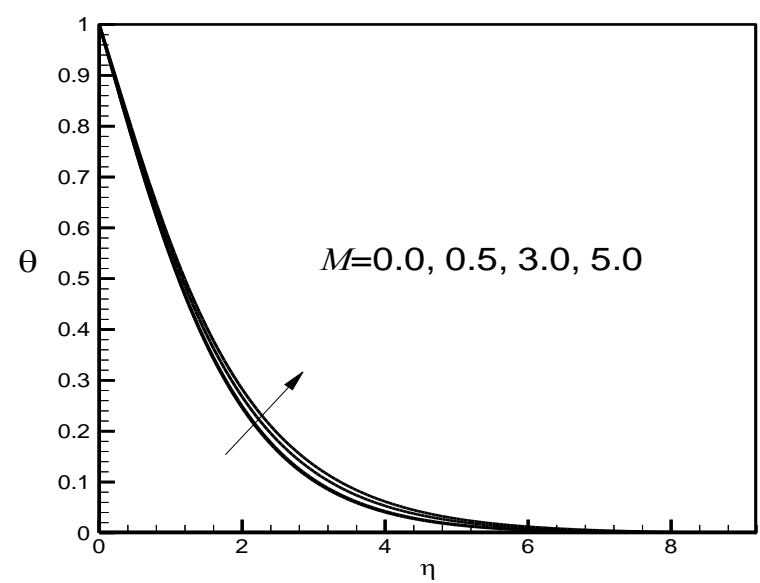

Fig. 19. Distribution of temperature for different values of $M$

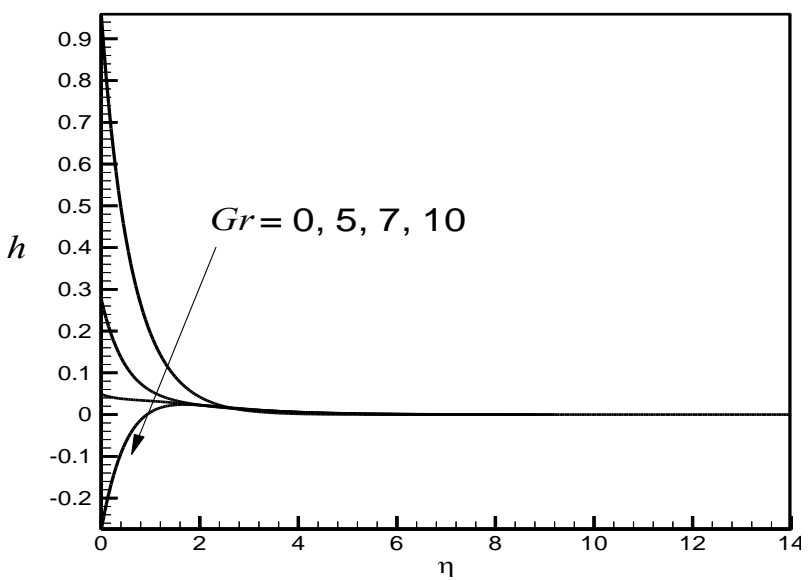

Fig. 21. Distribution of microrotation for different values of $\mathrm{Gr}$

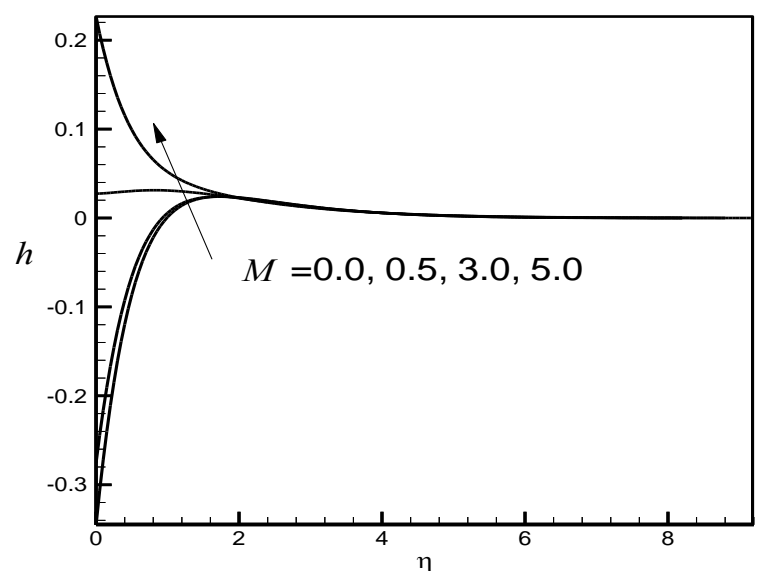

Fig. 18. Distribution of microrotation for different values of $M$

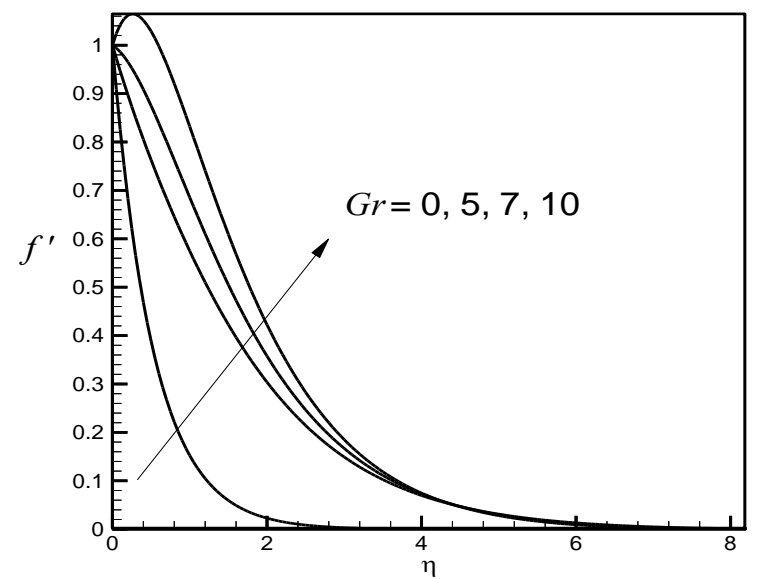

Fig. 20. Distribution of velocity for different values of $\mathrm{Gr}$

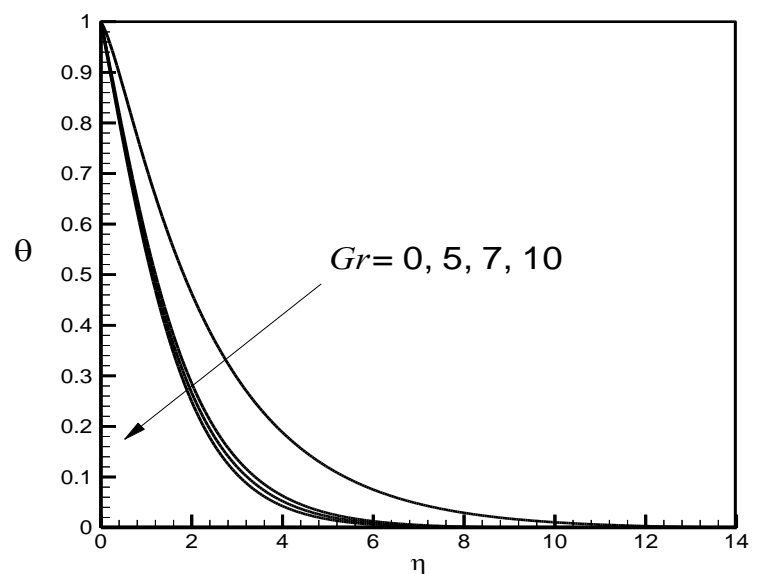

Fig. 22. Distribution of temperature for different values of $G r$ 


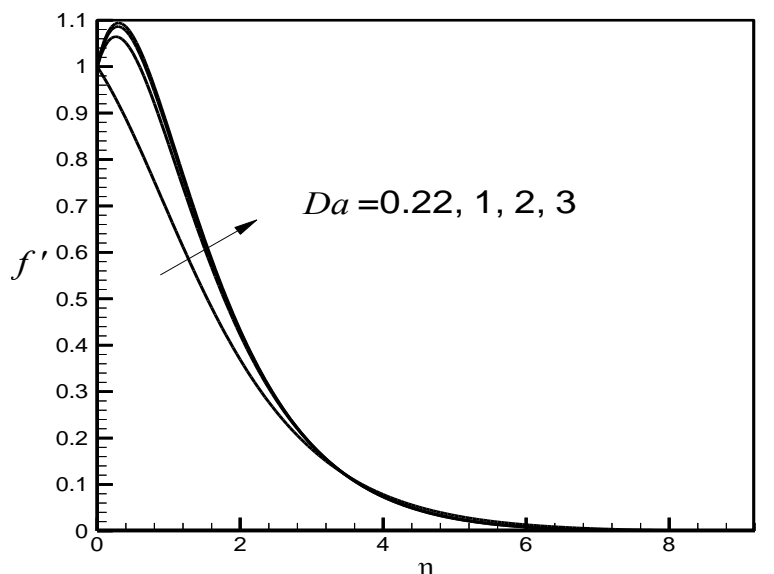

Fig. 23. Distribution of velocity for different values of $D a$

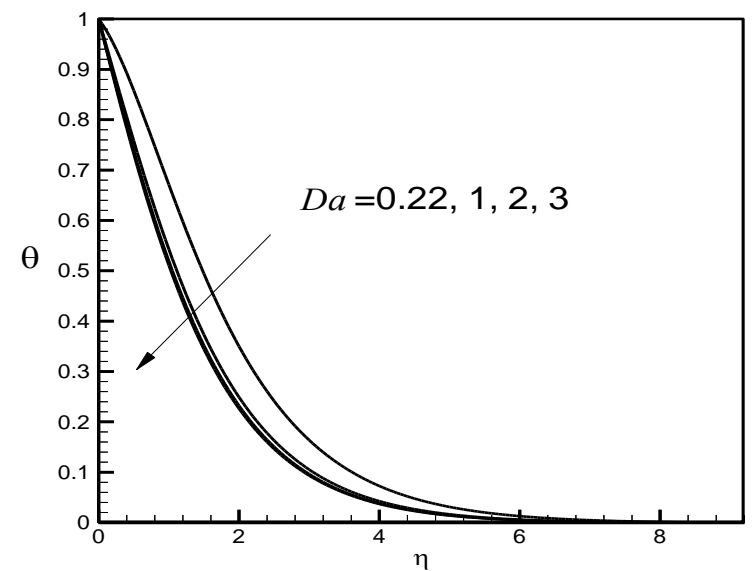

Fig. 24. Distribution of temperature for different values of $D a$

Figures 25, 26 encapsulate the variations of micropolar gas flow characteristics with Prandtl number $(\mathrm{Pr})$. Pr describes the relative influence of momentum and thermal diffusivities. Low $\operatorname{Pr}$ gases correspond to low-density gases, while high Pr values are associated with denser micropolar gases [51]. Since the present model is focused on gas flow as of air $\operatorname{Pr}=0.71$ [20] is considered in the modeling, unless otherwise indicated. Here we address the additional cases of micropolar liquids which may also be utilized in MHD power generators. Pralso defines the relative potency of momentum diffusion in the velocity boundary layer to heat diffusion in the thermal boundary layer. These phenomena are of the same order of magnitude for the special case when $\operatorname{Pr}=1$. For $\operatorname{Pr}<1$ physically corresponds that heat will transfer at an exceptionally faster rate than the momentum [52]. For $\operatorname{Pr}>1$, physically corresponds that momentum will diffuse faster than the heat through the regime. With greater Pr values Figures 25 and 26 reveals that velocity and temperature decreases. With higher $\mathrm{Pr}$, there is a sharp reduction in velocity and temperature profiles from the wall, whereas this reduction is much more gradual at low Pr. Similar results can be found in Wahid et al., [53]

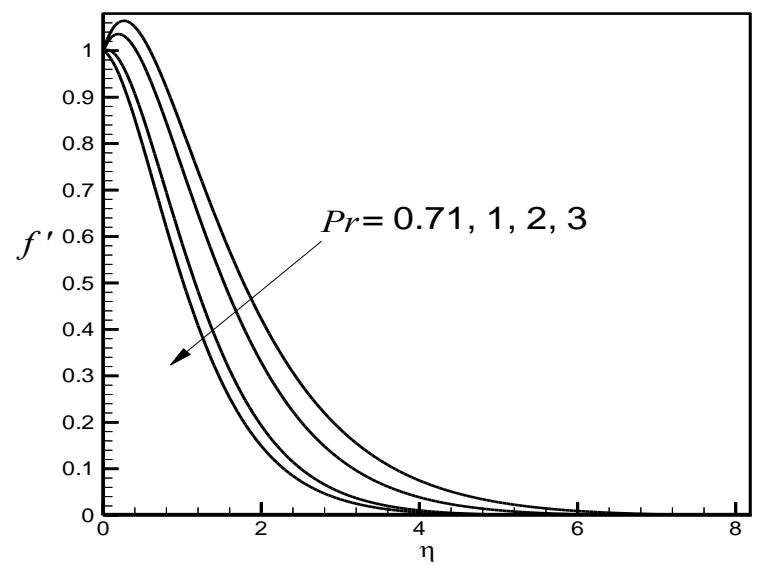

Fig. 25. Distribution of velocity for different values of $\mathrm{Pr}$

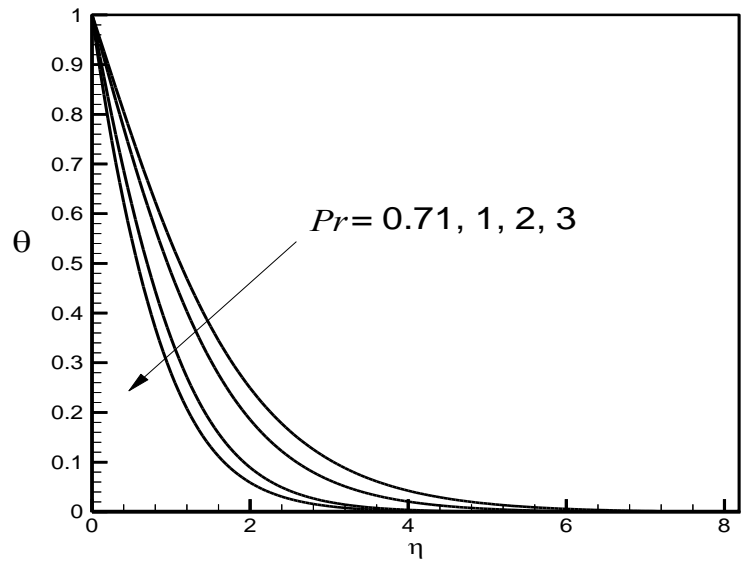

Fig. 26. Distribution of temperature for different values of $\mathrm{Pr}$

Figures 27, 28 illustrate the response in temperature and concentration functions to power-law index parameter $(\gamma)$. This parameter relates to the variation in wall temperature and wall concentration features in a single term in the energy equation and concentration equation i.e. Eqns. 
(13) and (14), namely $-(2 / n+1)\left[\gamma f^{\prime} \theta\right]$ and $-(2 / n+1)\left[\gamma f^{\prime} \phi\right]$. For $\gamma>0$ the wall temperature increases with distance from the leading edge. The case $\gamma=0$ corresponds to an isothermal wall. In the primitive Eq. (8), $\gamma$ arises in the wall thermal boundary condition as $T=T_{w}\left(=T_{\infty}+A x^{\gamma}\right)$ and wall solutal boundary condition as $C=C_{w}\left(=C_{\infty}+D x^{\gamma}\right)$. However, following normalization of the equations, it is featured in the thermal and solutal transport equations (13) and (14). Temperature and concentration are observed to be suppressed with positive $\gamma$ values.

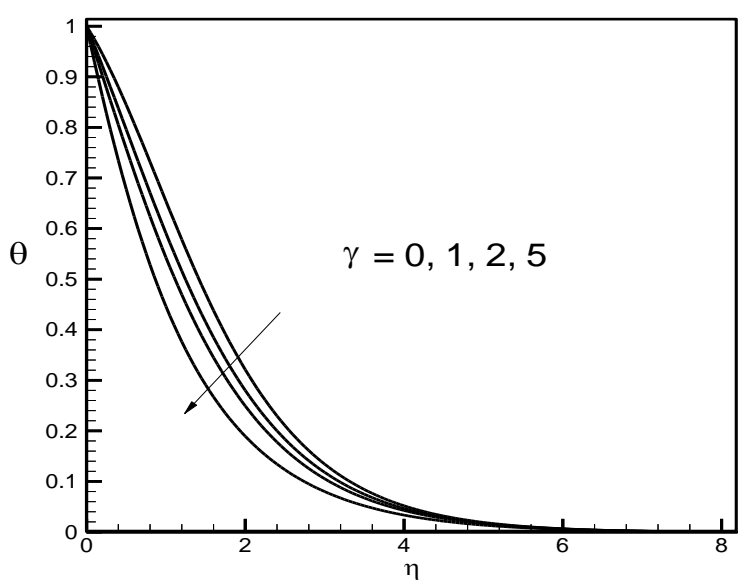

Fig. 27. Distribution of temperature for different values of $\gamma$

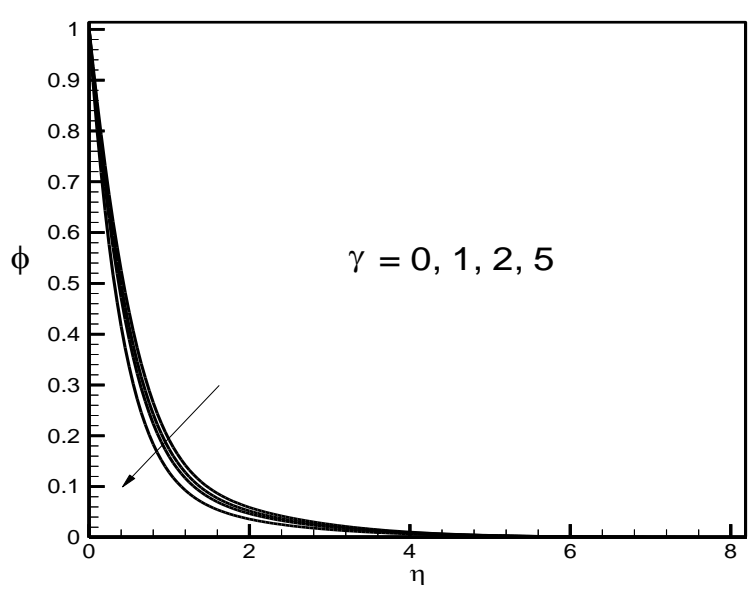

Fig. 28. Distribution of concentration for different values of $\gamma$

Figures 29, 30 illustrates the impact of the radiation effect on velocity and temperature contours. The radiation-conduction parameter, $R=\kappa \kappa_{1} / 4 \sigma_{1} T_{\infty}^{3}$ defined as the relative contribution of thermal radiation heat transfer to thermal conduction heat transfer. This is an equitable approximation for optically thick micropolar flow, as considered here. This approximation, however, cannot simulate variation in optical thickness, which requires a more sophisticated flux model [52]. In the present simulations, we enclose attention to the special case of $R>1$ (thermal radiation dominates over thermal conduction). Thus, thermal radiative flux is substantial as disclosed in Figure 29. It is seen that there is strong retardation in the linear velocity with increasing $R$ values. As the flow initiate and strengthen the thermal diffusion but impede momentum diffusion.

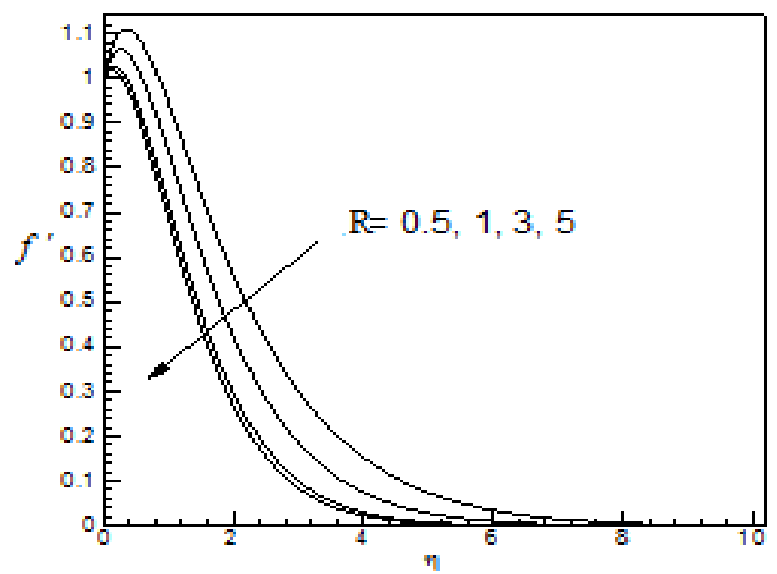

Fig. 29. Distribution of velocity for different values of $R$

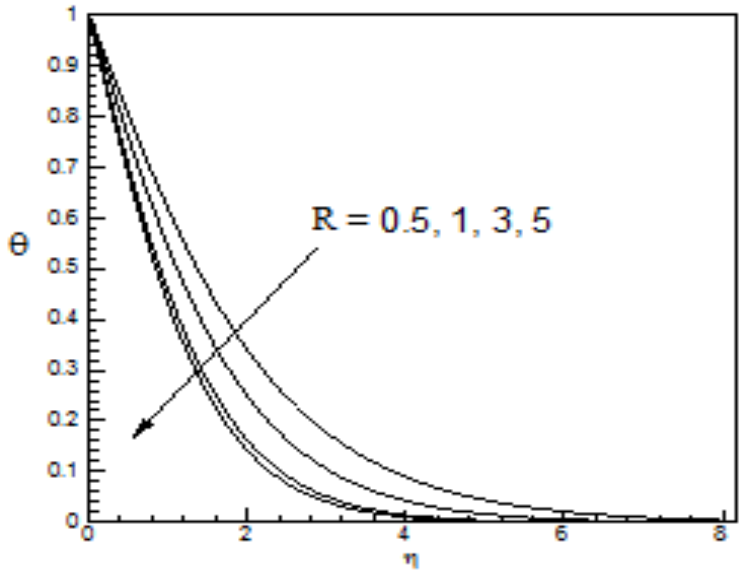

Fig. 30. Distribution of temperature for different values of $\mathrm{R}$ 
This advantage to an increase in momentum boundary layer thickness. Increasing the radiationconduction parameter is also found to decrease temperatures in the boundary layer (Figure 30). Thermal boundary layer thickness is therefore reduced with greater values of $R$. Physically speaking, an increase in radiation parameter means the release of heat energy from the flow region, and hence the boundary layer thickness becomes thinner.

Figures 31, 32 illustrate the response in translational velocity and temperature functions to Eckert number $(E c) . E c$ expresses the relationship between the kinetic energy in the flow and the enthalpy difference (Gschwendtner [54]). It, therefore, represents the conversion of kinetic energy into internal energy by work done against the viscous fluid stresses. Although this parameter is often used in a high-speed compressible flow, for example in rocket aerodynamics at very altitude, it has significance in high-temperature incompressible flows, which are encountered in energy systems, etc [55]. Positive Eckert number implies cooling of the wall and therefore a transfer of heat to the fluid. Convection is enhanced. It is seen that the translational velocity flow is accelerated (Figure 31). The proportion of kinetic energy dissipated as heat manifests also with a strong elevation in temperature (Figure 32). Thermal boundary layer thickness is therefore considerably increased with higher Eckert number.

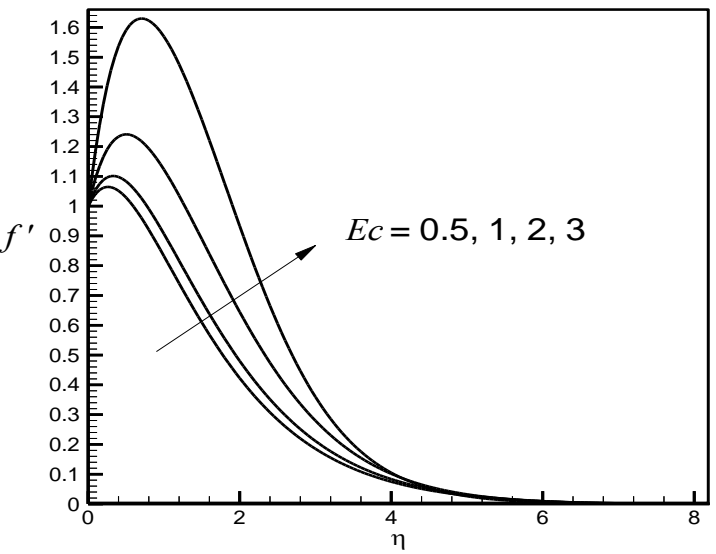

Fig. 31. Distribution of velocity for different values of $E c$

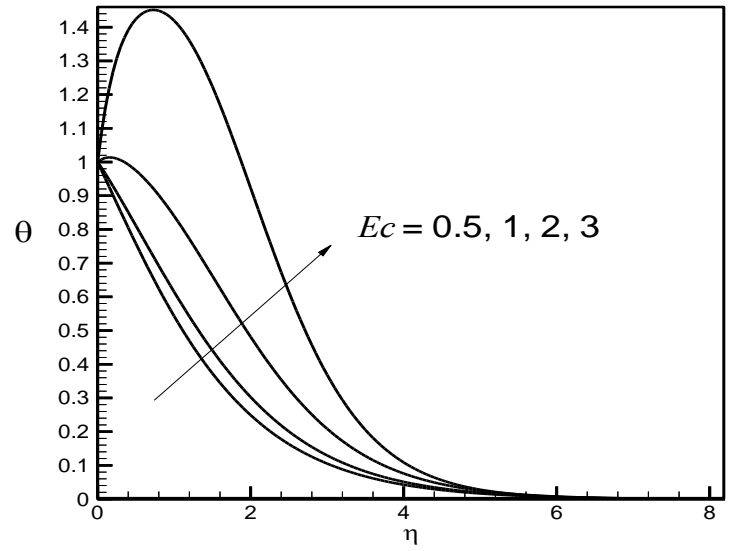

Fig. 32. Distribution of temperature for different values of $E c$

Figure 33 illustrates the response of concentration profiles to different values of the Schmidt number $(S c)$. Schmidt number is a fundamental parameter in species diffusion (mass transfer), which describes the ratio of the momentum to the molecular (species) diffusivity i.e. $S c=v / D_{m}$ [56]. The Schmidt number, therefore, quantifies the relative effectiveness of momentum and mass transport by diffusion in the hydrodynamic (velocity) and concentration (species) boundary layers. It is observed that as the Schmidt number increases concentration decrease. The associated decrease in species diffusivity results in the less vigorous mass transfer which reduces concentration levels and also depletes the concentration boundary layer thickness.

Figures 34-37 depict the impact of Dufour number $(\mathrm{Du})$ and Soret number $(\mathrm{Sr})$ on the temperature and concentration function, respectively. The Dufour number signifies the contribution of the concentration gradient to the thermal energy flux in the flow [56]. The influence of Soret number defines the effect of temperature gradient inducing significant mass diffusion effects [57]. Figures 34 and 35 show non-dimensional temperature and concentration profiles for different values of $D u$. As $D u$ increases, the temperature of the fluid increases, while, the concentration of the fluid decelerates in the boundary layer regime. 


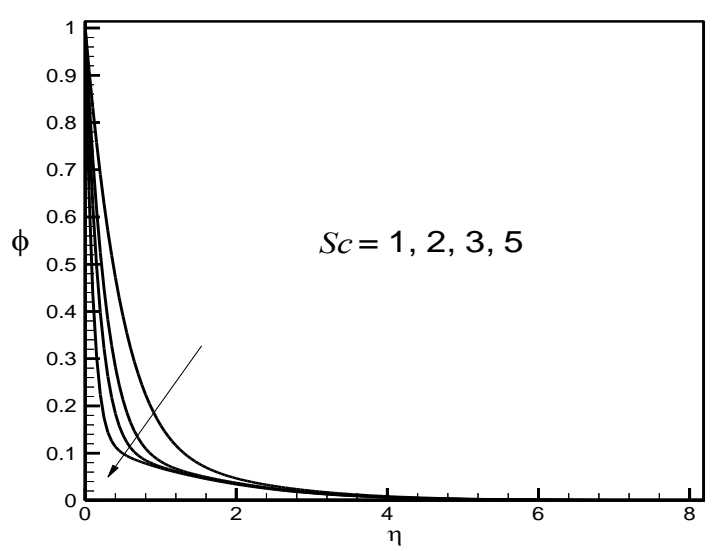

Fig. 33. Distribution of concentration for different values of $S c$

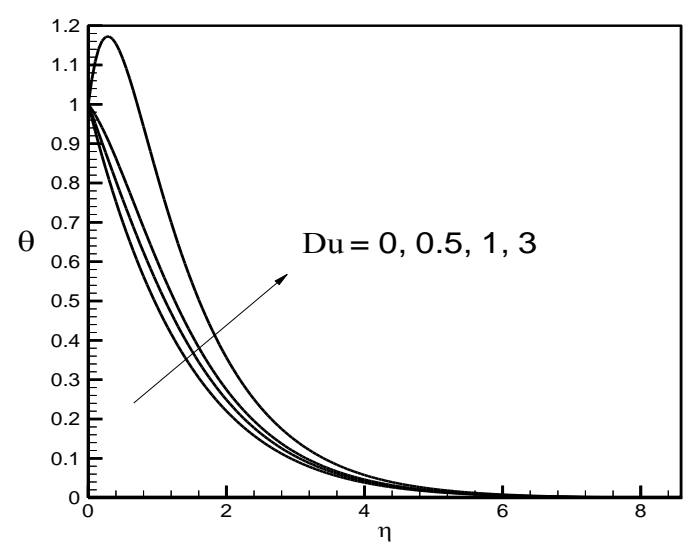

Fig. 34. Distribution of temperature for different values of Du

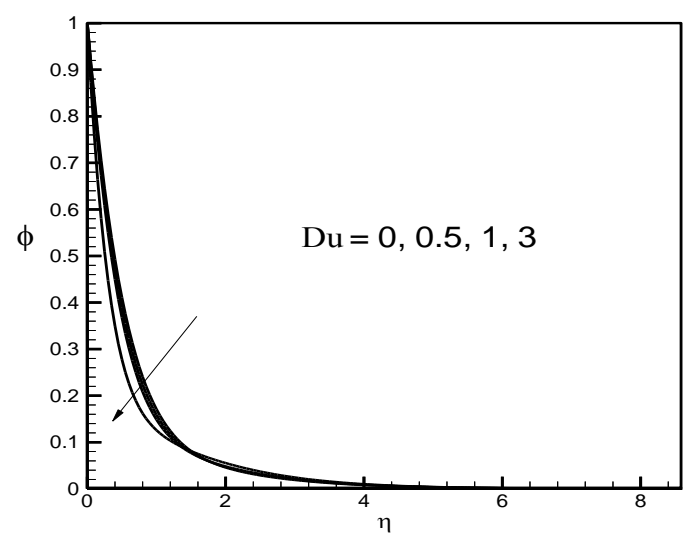

Fig. 35. Distribution of concentration for different values of $\mathrm{Du}$

Figures 36 and 37 illustrate the temperature and concentration distributions for different values of $\mathrm{Sr}$. It is seen that by increasing Soret number, the temperature distributions decrease, while, the concentration profiles increase in the flow field. This is because of the fact that the diffusive species with higher values of Soret parameter $(\mathrm{Sr})$ has the tendency of increasing concentration profiles.

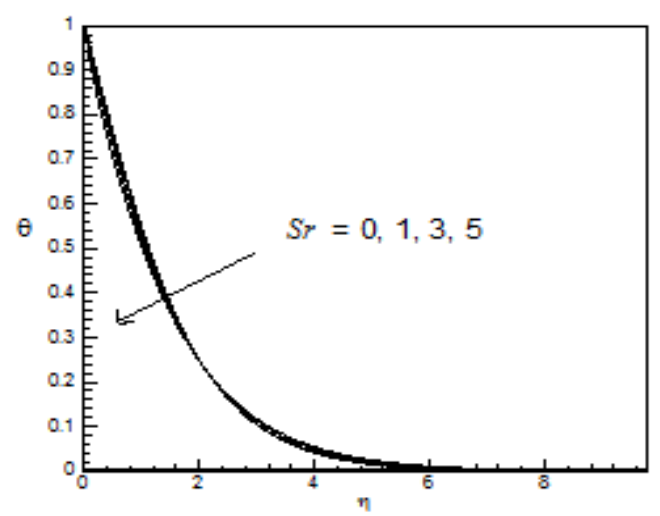

Fig. 36. Distribution of temperature for different values of $\mathrm{Sr}$

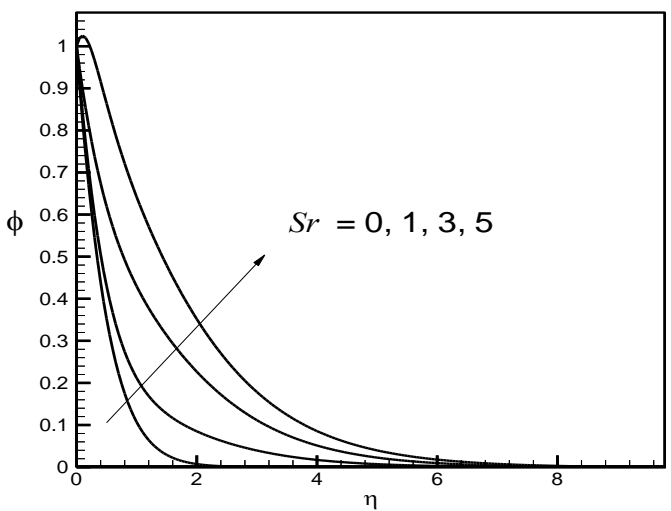

Fig. 37. Distribution of concentration for different values of $\mathrm{Sr}$

The concentration of the fluid increases due to the enhanced thermal diffusion rate. Physically, a high Soret number stands for a larger temperature difference and precipitous gradient. Here $\Delta=0$ 
gives the result for viscous incompressible fluid these trends can be seen in Shamshuddin et al., [58]. From this, it is observed that the velocity of the Newtonian fluid is greater than that of the micropolar fluid. Hence, the temperature and concentration distributions are more influenced by the values of $\mathrm{Du}$ and $\mathrm{Sr}$.

\section{Conclusions}

In the present work, cross-diffusion, viscous dissipation and thermal radiation effects on the hydromagnetic stream of magnetic micropolar fluid due to a horizontal stretching sheet immersed in a non-Darcy porous medium have been analyzed numerically. Nachtsheim-Swigert iteration technique along with the sixth order Runge-Kutta integration scheme is utilized for a system of coupled nonlinear, non-dimensional ordinary differential equations, which are transformed by similarity variables subjected to power-law thermal and solutal boundary conditions. Results on the influence of several parameters (e.g. $\Delta, f_{w}, n, M, G r, D a, \operatorname{Pr}, \gamma, R, E c, S c, D u$ and $S r$ ) on velocity, microrotation, temperature, and concentration are presented. Results show that the micropolar fluid velocity decreases with the increase of vortex viscosity ratio parameter, suction parameter and radiation parameter. However, the velocity increases by increasing the magnetic field parameter, Grashof number, Darcy number, and Eckert number. Microrotation distributions of the micropolar fluid augment for vortex viscosity ratio parameter, suction parameter and magnetic field parameter are elevated while the reverse trend is observed in case of Grashof number. Temperature distributions of the micropolar fluid escalate for vortex viscosity ratio parameter, suction parameter, magnetic field parameter, and Dufour number while the reverse trend is observed in case of Grashof number, Darcy number, Prandtl number, Eckert number, Soret number and thermal radiation parameter. Concentration profiles of the magnetic micropolar fluid putrefy for rising values of the suction, constant parameter, Schmidt number, and Dufour number. However, concentration distributions increase with the increase of Soret number and surface nonlinearity parameter. The present simulations have been confined to steady-state flow. Further investigation will consider transient model. It is envisaged that further interest in more realistic fluid flow in Artificial Cancellous bone prediction [59]. Effects in this regard are underway and will be communicated imminently.

\section{Acknowledgement}

The author would like to acknowledge the support from the Fundamental Research Grant Scheme (FRGS) under a grant number of FRGS/1/2018/STG06/UNIMAP/02/3 from the Ministry of Education Malaysia.

\section{References}

[1] Lukaszewicz, Grzegorz. Micropolar fluids: theory and applications. Springer Science \& Business Media, 1999. https://doi.org/10.1007/978-1-4612-0641-5 5

[2] Rajagopal, Kumbakonam R., T. Y. Na, and A. S. Gupta. "Flow of a viscoelastic fluid over a stretching sheet." Rheologica Acta 23, no. 2 (1984): 213-215. https://doi.org/10.1007/BF01332078

[3] Eringen, A. Cemal. "Theory of micropolar fluids." Journal of Mathematics and Mechanics (1966): 1-18. https://doi.org/10.1512/iumj.1967.16.16001

[4] Raman, N., S. Sudharsan, and K. Pothiraj. "Synthesis and structural reactivity of inorganic-organic hybrid nanocomposites-a review." Journal of Saudi Chemical Society 16, no. 4 (2012): 339-352. https://doi.org/10.1016/j.jscs.2011.01.012

[5] Crane, L.J. "Flow past a stretching sheet." Zeitschrift für Angewandte Mathematik und Physik (ZAMP) 21, no. 4 (1970): 645-647. https://doi.org/10.1007/BF01587695 
[6] Vajravelu, Kuppalapalle, and R. Van Gorder. Nonlinear flow phenomena and homotopy analysis. Berlin: Springer, 2013.

https://doi.org/10.1007/978-3-642-32102-3

[7] Aydin, Orhan, and loan Pop. "Natural convection from a discrete heater in enclosures filled with a micropolar fluid." International journal of engineering science 43, no. 19-20 (2005): 1409-1418. https://doi.org/10.1016/j.ijengsci.2005.06.005

[8] Bachok, N., A. Ishak, and R. Nazar. "Flow and heat transfer over an unsteady stretching sheet in a micropolar fluid with prescribed surface heat flux." International journal of Mathematical models and Methods in applied Sciences 4, no. 3 (2010): 167-176.

[9] Char, Ming-I., and Cheng-Long Chang. "Effect of wall conduction on natural convection flow of micropolar fluids along a flat plate." International journal of heat and mass transfer 40, no. 15 (1997): 3641-3652. https://doi.org/10.1016/S0017-9310(97)00006-9

[10] El-Arabawy, Hassan AM. "Effect of suction/injection on the flow of a micropolar fluid past a continuously moving plate in the presence of radiation." International Journal of Heat and Mass Transfer 46, no. 8 (2003): 1471-1477. https://doi.org/10.1016/S0017-9310(02)00320-4

[11] Hassanien, I. A., A. H. Essawy, and N. M. Moursy. "Natural convection flow of micropolar fluid from a permeable uniform heat flux surface in porous medium." Applied mathematics and computation 152, no. 2 (2004): 323-335. https://doi.org/10.1016/S0096-3003(03)00556-3

[12] Hayat, T., M. Nawaz, and S. Obaidat. "Axisymmetric magnetohydrodynamic flow of micropolar fluid between unsteady stretching surfaces." Applied Mathematics and Mechanics 32, no. 3 (2011): 361-374. https://doi.org/10.1007/s10483-011-1421-8

[13] Ishak, Anuar, Roslinda Nazar, and Ioan Pop. "The Schneider problem for a micropolar fluid." Fluid dynamics research 38, no. 7 (2006): 489-502.

https://doi.org/10.1016/i.fluiddyn.2006.03.004

[14] Karim, M. Enamul, M. A. Samad, and Md Abdus Sattar. "Steady MHD Free Convection Flow with Thermal Radiation Past a Vertical Porous Plate Immersed in a Porous Medium." Research Journal of Mathematics and Statistics 3, no. 4 (2011): 141-147.

[15] Lok, Yian Yian, Norsarahaida Amin, and loan Pop. "Unsteady boundary layer flow of a micropolar fluid near the rear stagnation point of a plane surface." International Journal of Thermal Sciences 42, no. 11 (2003): 995-1001. https://doi.org/10.1016/S1290-0729(03)00079-6

[16] Lok, Y. Y., I. Pop, and Ali J. Chamkha. "Non-orthogonal stagnation-point flow of a micropolar fluid." International Journal of Engineering Science 45, no. 1 (2007): 173-184. https://doi.org/10.1016/i.ijengsci.2006.04.016

[17] Motsa, Sandile Sydney, and Stanford Shateyi. "The effects of chemical reaction, hall, and ion-slip currents on MHD micropolar fluid flow with thermal diffusivity using a novel numerical technique." Journal of Applied Mathematics 2012 (2012). https://doi.org/10.1155/2012/689015

[18] Nazar, Roslinda, Anuar Ishak, and loan Pop. "Unsteady boundary layer flow over a stretching sheet in a micropolar fluid." International Journal of Mathematical, Physical and Engineering Sciences 2, no. 3 (2008): 161-165.

[19] Rahman, Mohammad M., M. A. Rahman, M. A. Samad, and M. S. Alam. "Heat transfer in a micropolar fluid along a non-linear stretching sheet with a temperature-dependent viscosity and variable surface temperature." International Journal of Thermophysics 30, no. 5 (2009): 1649-1670. https://doi.org/10.1007/s10765-009-0656-5

[20] Rahman, M. M., M. J. Uddin, and A. Aziz. "Convective Flow of Micropolar Fluid in a Porous Medium with Variable Electric Conductivity, Surface Heat Flux and NonUniform Heat Source (or Sink)." International Journal of Energy \& Technology 25, no. 2 (2010): 1-18.

[21] Takhar, Harmindar S., R. Bhargava, R. S. Agrawal, and A. V. S. Balaji. "Finite element solution of micropolar fluid flow and heat transfer between two porous discs." International journal of engineering science 38, no. 17 (2000): 1907-1922.

https://doi.org/10.1016/S0020-7225(00)00019-7

[22] Ishak, Anuar. "Thermal boundary layer flow over a stretching sheet in a micropolar fluid with radiation effect." Meccanica 45, no. 3 (2010): 367-373. https://doi.org/10.1007/s11012-009-9257-4

[23] Ahmad, Kartini, Anuar Ishak, and Roslinda Nazar. "Micropolar fluid flow and heat transfer over a nonlinearly stretching plate with viscous dissipation." Mathematical Problems in Engineering 2013 (2013). https://doi.org/10.1155/2013/257161 
[24] Prasad, K. V., H. Vaidya, and K. Vajravelu. "Convective heat transfer in a micropolar fluid over an unsteady stretching surface." International Journal of Applied Mechanics and Engineering 21, no. 2 (2016): 407-422. https://doi.org/10.1515/ijame-2016-0025

[25] Rawat, S., S. Kapoor, and R. Bhargava. "MHD Flow Heat and Mass Transfer of Micropolar Fluid over a Nonlinear Stretching Sheet with Variable Micro Inertia Density, Heat Flux and Chemical Reaction in a Non-darcy Porous Medium." Journal of Applied Fluid Mechanics 9, no. 1 (2016). https://doi.org/10.18869/acadpub.jafm.68.224.23957

[26] Shamshuddin, M. D., Thumma Thirupathi, and P. V. Satya Narayana. "Micropolar fluid flow induced due to a stretching sheet with heat source/sink and surface heat flux boundary condition effects." Journal of Applied and Computational Mechanics 5, no. 5 (2019): 816-826.

[27] Shamshuddin, M. D., Thirupathi Thumma, and S. R. Mishra. "Thermo-Solutal Chemically Reacting Micropolar Fluid Past a Permeable Stretching Porous Sheet." In Defect and Diffusion Forum, vol. 392, pp. 42-59. Trans Tech Publications Ltd, 2019. https://doi.org/10.4028/www.scientific.net/DDF.392.42

[28] Eid, Mohamed R., Kasseb L. Mahny, Taseer Muhammad, and Mohsen Sheikholeslami. "Numerical treatment for Carreau nanofluid flow over a porous nonlinear stretching surface." Results in physics 8 (2018): 1185-1193. https://doi.org/10.1016/j.rinp.2018.01.070

[29] Eid, Mohamed R., K. L. Mahny, Amanullah Dar, and Taseer Muhammad. "Numerical study for Carreau nanofluid flow over a convectively heated nonlinear stretching surface with chemically reactive species." Physica A: Statistical Mechanics and its Applications 540 (2020): 123063.

https://doi.org/10.1016/i.physa.2019.123063

[30] Al-Hossainy, Ahmed F., Mohamed R. Eid, and Mohamed Sh Zoromba. "SQLM for external yield stress effect on 3D MHD nanofluid flow in a porous medium." Physica Scripta 94, no. 10 (2019): 105208. https://doi.org/10.1088/1402-4896/ab2413

[31] Eid, Mohamed R. "Effects of NP Shapes on Non-Newtonian Bio-Nanofluid Flow in Suction/Blowing Process with Convective Condition: Sisko Model." Journal of Non-Equilibrium Thermodynamics 1, no. ahead-of-print (2019). https://doi.org/10.1515/inet-2019-0073

[32] Lahmar, Sihem, Mohamed Kezzar, Mohamed R. Eid, and Mohamed Rafik Sari. "Heat transfer of squeezing unsteady nanofluid flow under the effects of an inclined magnetic field and variable thermal conductivity." Physica $A$ : Statistical Mechanics and its Applications 540 (2020): 123138.

https://doi.org/10.1016/i.physa.2019.123138

[33] Eckert, Ernst Rudolf Georg, and Robert M. Drake Jr. "Analysis of heat and mass transfer." (1987). https://doi.org/10.1002/aic.690180342

[34] Hayat, T., and F. A. Hendi. "Thermal-diffusion and diffusion-thermo effects on MHD three-dimensional axisymmetric flow with Hall and ion-slip currents." Journal of American Science 8, no. 1 (2012): 284-294.

[35] Makinde, Oluwole Daniel, and Philip Oladapo Olanrewaju. "Unsteady mixed convection with Soret and Dufour effects past a porous plate moving through a binary mixture of chemically reacting fluid." Chemical Engineering Communications 198, no. 7 (2011): 920-938. https://doi.org/10.1080/00986445.2011.545296

[36] Shateyi, Stanford, Sandile Sydney Motsa, and Precious Sibanda. "The effects of thermal radiation, hall currents, soret, and dufour on MHD flow by mixed convection over a vertical surface in porous media." Mathematical Problems in Engineering 2010 (2010). https://doi.org/10.1155/2010/627475

[37] Srinivasacharya, D. and Kaladhar, Kolla." Soret and Dufour effects on mixed convection flowof couple stress fluid in a non-darcy porous medium with heat and mass fluxes." Journal of Porous Media 17, no. 2 (2014): 93-101. https://doi.org/10.1615/JPorMedia.v17.i2.10

[38] Shamshuddin, M.D. and Siva Reddy S. "Free convection from a rotating vertical porous plate in a dissipative micropolar fluid with cross-diffusion effects." Modeling, Measurement and Control B 86, no.3 (2018): 627-657. https://doi.org/10.18280/mmc b.860304

[39] Shamshuddin, M. D., A. J. Chamkha, M. C. Raju, and Thirupathi Thumma. "Computation of unsteady mhd mixed convective heat and mass transfer in dissipative reactive micropolar flow considering sorte and dufour effects." Frontiers in Heat and Mass Transfer (FHMT) 10 (2018). https://doi.org/10.5098/hmt.10.15

[40] Anjali Devi, S.P. and B. Ganga. "Dissipation effects on MHD nonlinear flow and heat transfer past a porous surface with prescribed heat flux." (2010): 1-6. https://doi.org/10.36884/jafm.3.01.11873 
[41] Singh, P. K., and Jai Singh. "MHD flow with viscous dissipation and chemical reaction over a stretching porous plate in porous medium." International Journal of Engineering Research and Applications 2, no. 2 (2012): 1556-1564.

[42] Uwanta, I. J., B. Y. Isah, and M. O. Ibrahim. "Viscoelastic fluid flow past an infinite vertical plate with heat dissipation." International Journal of Computer Applications 36, no. 2 (2011).

[43] Al-Hadhrami, A. K., L. Elliott, and D. B. Ingham. "A new model for viscous dissipation in porous media across a range of permeability values." Transport in porous media 53, no. 1 (2003): 117-122. https://doi.org/10.1023/A:1023557332542

[44] Bhargava, R., Rajesh Sharma, and O. Anwar Bég. "A numerical solution for the effect of radiation on micropolar flow and heat transfer past a horizontal stretching sheet through porous medium." In Proceedings of the 5th IASME/WSEAS International Conference on Continuum Mechanics (CM'10). 2010.

[45] Sparrow, E. M., and R. D. Cess. "Radiation Heat Transfer, Augmented edition, Hemisphere Publ." Crop., Washington, $D C$ (1978).

[46] Bég, O. Anwar, Joaquín Zueco, Swapan Kumar Ghosh, and Alireza Heidari. "Unsteady magnetohydrodynamic heat transfer in a semi-infinite porous medium with thermal radiation flux: analytical and numerical study." Advances in Numerical Analysis 2011 (2011). https://doi.org/10.1155/2011/304124

[47] Beg, O. Anwar, M. Ferdows, M. Enamul Karim, M. Maruf Hasan, T. A. Bég, M. D. Shamshuddin, and A. Kadir. "Computation of non-isothermal thermo-convective micropolar fluid dynamics in a Hall MHD generator system with non-linear distending wall." International Journal of Applied and Computational Mathematics 6, no. 2 (2020): 1-44.

https://doi.org/10.1007/s40819-020-0792-y

[48] Sparrow, E. M., and H. S. Yu. "Local non-similarity thermal boundary-layer solutions." (1971): 328-334. https://doi.org/10.1115/1.3449827

[49] Pantokratoras, Asterios. "Four usual errors made in investigation of boundary layer flows." Powder Technology 353 (2019): 505-508. https://doi.org/10.1016/i.powtec.2019.05.060

[50] Nachtsheim, Philip R., and Paul Swigert. "Statisfaction of asymptotic boundary conditions in numerical solution of systems of nonlinear equations of boundary-layer type." PhD Thesis, NASA TN D-3004, Washington, D.C. 1965.

[51] Cebeci, Tuncer, and Peter Bradshaw. Physical and computational aspects of convective heat transfer. Springer Science \& Business Media, 2012.

https://doi.org/10.1007/978-3-662-02411-9

[52] Kuharat, Sireetorn, O. Anwar Bég, Ali Kadir, and M. D. Shamshuddin. "Computational study of heat transfer in solar collectors with different radiative flux models." Heat Transfer-Asian Research 48, no. 3 (2019): 1002-1031. https://doi.org/10.1002/htj.21418

[53] Wahid, Nur Syahirah, Mohd Ezad Hafidz Hafidzuddin, Norihan Md Arifin, Mustafa Turkyilmazoglu, and Nor Aliza Abd Rahmin. "Magnetohydrodynamic (MHD) Slip Darcy Flow of Viscoelastic Fluid Over A Stretching Sheet and Heat Transfer with Thermal Radiation and Viscous Dissipation." CFD Lettes 12, no.1 (2020): 1-12.

[54] Gschwendtner, M. A. "The Eckert number phenomenon: Experimental investigations on the heat transfer from a moving wall in the case of a rotating cylinder." Heat and mass transfer 40, no. 6-7 (2004): 551-559. https://doi.org/10.1007/s00231-003-0437-9

[55] Bég, O. Anwar, Bettina Islam, M. D. Shamshuddin, and Tasveer A. Bég. "Computational fluid dynamics analysis of moisture ingress in aircraft structural composite materials." Arabian Journal for Science and Engineering 44, no. 9 (2019): 7809-7831.

https://doi.org/10.1007/s13369-019-03917-4

[56] Bergman, Theodore L., Frank P. Incropera, David P. DeWitt, and Adrienne S. Lavine. Fundamentals of heat and mass transfer. John Wiley \& Sons, 2011.

[57] Partha, M. K., P. V. S. N. Murthy, and G. P. Raja Sekhar. "Soret and Dufour effects in a non-Darcy porous medium." (2006): 605-610. https://doi.org/10.1115/1.2188512

[58] MD, Shamshuddin, Siva Reddy Sheri, and O. Anwar Bég. "Oscillatory dissipative conjugate heat and mass transfer in chemically reacting micropolar flow with wall couple stress: A finite element numerical study." Proceedings of the Institution of Mechanical Engineers, Part E: Journal of Process Mechanical Engineering 233, no. 1 (2019): 48-64. https://doi.org/10.1177/0954408917743372

[59] Yamina, Elsa Syuhada Abdull, and CS Nor Azwadi. "Prediction of Fluid Flow in Artificial Cancellous Bone." Journal of Advanced Research in Materials Science 3, no. 1 (2014): 8-14. 